\title{
LA CARRERA DE PSICOLOGÍA EN LA UNIVERSIDAD DE LA FRONTERA: UNA EXPERIENCIA DE ACREDITACIÓN
}

Reginaldo Zurita* 


\section{RESUMEN}

El artículo describe el proceso de acreditación de la carrera de Psicología en la Universidad de La Frontera de Temuco, con la Comisión Nacional de Acreditación de Pregrado (CNAP). En el marco de las orientaciones y acciones de esta universidad sobre aseguramiento de la calidad -tanto institucional como de carreras-, se identifican y describen las fases de este proceso (objetivos, organización, actividades, redacción y presentación del Informe de Autoevaluación). A modo de corolario, y sobre la base de considerar este proceso experimental de acreditación como una apertura de oportunidades y, por ende, de aprendizaje para los actores involucrados, se enuncian, sintéticamente, los juicios de las autoridades universitarias y, obviamente, de las personas directamente involucradas con la carrera de Psicología de esta universidad.

\section{ABSTRACT}

The paper describes the accreditation process for the Psychology study program at the Universidad de La Frontera at Temuco with the National Commission for Undergraduate Accreditation (CNAP). The article describes the phases involved in this process (objectives, organization, activities, drafting and submission of the Self-assessment Report) in the framework of the approaches and actions carried out by the university on quality assurance -both in institutional terms as well as with regard to the study programs. As a corollary, and by considering this experimental accreditation process an opening up of opportunities and therefore a chance for learning for the actors involved, the paper provides a summarized version of the views of the university authorities and, evidently of the individuals involved directly in the Psychology study program at the university. 


\author{
LA CARRERA DE PSICOLOGÍA EN LA \\ UNIVERSIDAD DE LA FRONTERA: \\ UNA EXPERIENCIA DE ACREDITACIÓN
}

Una premonición

Se suele afirmar que para comprender los problemas del presente es aconsejable volver a los clásicos. En un discurso pronunciado en 1962, don Juan Gómez Millas, ex rector de la Universidad de Chile y ex ministro de Educación, advertía:

"Para que el diálogo racional sea fecundante, es necesaria la autocrítica en la universidad. Es bueno que los que en ella actúan como estudiantes o como docentes sepan cómo está en cada momento la universidad; qué fue de ella antes y qué debe llegar a ser mañana [...] Las universidades revisan constantemente sus métodos de trabajo, el cumplimiento correcto de sus fines y la calidad del trabajo docente que ejecutan sus profesores.

Ningún argumento de estabilidad funcionaria puede destruir o debilitar esta actitud, porque las universidades no son lugares para el descanso, sino de inquietud y cambio constante; todo conformismo en ellas lleva a la parálisis de la sociedad a la cual sirve y todos los que tenemos una pequeña o grande responsabilidad en la universidad debemos ser insensibles con la popularidad barata y transitoria que consiste en ser tolerante con los defectos y malos hábitos que en ella se manifiestan o con la calidad del personal que debe darle vida y aliento. Es un deber de todos nosotros estar atentos a las críticas honradas de los estudiantes y de la sociedad, so pena de periclitar en la decadencia y la esterilidad (Gómez Millas, 1986)." 
Recuérdese además que el Presidente Nixon, en un mensaje al Congreso de los EE.UU., había declarado: "Si los sesenta fueron los años de la innovación educativa, los setenta serán designados como la década del accountability...", es decir, de la rendición de cuentas. Hacia 1950 los economistas occidentales comenzaron a ocuparse de los recursos humanos y a mirar con nuevos ojos el campo de la educación. Ya no la perciben como un sector no productivo de la economía que absorbe gastos irreparables, sino como una inversión esencial para el crecimiento económico (Coombs, 1970).

Sin embargo, en la lógica de la rendición de cuentas, se sacralizan a partir de entonces las técnicas de formulación de objetivos operacionales en términos de conducta observable y medible del alumno, vehiculizadas por la tecnología educativa que emerge e inunda este ámbito durante la década de los ochenta. Fue tal la convicción, que se llegó a sostener que la solución de los problemas educacionales dependía de que los profesores formularan sus objetivos de aprendizaje en términos de la conducta observable y medible de los estudiantes. Se trataba de que el $90 \%$ de los alumnos lograra el $90 \%$ de los objetivos (Block, 1975).

Premonitorio este sabio maestro chileno, porque el sentido fundamental de sus palabras tiene que ver con la fe pública. Hasta 1980 las universidades chilenas estaban "socialmente" acreditadas, es decir, garantizaban la fe pública en términos de su credibilidad social: sus profesionales ejercían su profesión con autoridad. Con la reforma de los ochenta, que se traduce en una diversificación vertical y horizontal de la educación superior y un crecimiento explosivo, surge la necesidad de asegurar la calidad, de crear instancias que aseguren la fe pública, proceso que se formaliza en lo que devino en llamarse "Sistema Nacional de Aseguramiento de la Calidad de la Educación Superior", recientemente aprobado por el Senado, y cuyos objetivos específicos son:

a) dar garantía pública de la calidad de las instituciones de educación superior y de los programas que imparten;

b) promover y apoyar el mejoramiento continuo y sistemático de las instituciones de educación superior y de los programas que ofrecen; 
c) establecer una instancia de coordinación del conjunto de funciones y actividades que apuntan al desarrollo de la calidad de la educación superior;

d) mejorar las condiciones para el desarrollo de una educación superior capaz de responder a estándares de validez nacional e internacional.

Orientaciones para la acción

En este marco, la Universidad de La Frontera, seria y responsablemente, inició el trabajo para la acreditación institucional y para sus carreras de pregrado y posgrado.

La política de mejoramiento de la calidad de la educción superior en Chile ha ido a la par del desarrollo de instrumentos orientados al fortalecimiento de las instituciones. Entre ellos, los que han alcanzado mayor relevancia e impacto en los planes de desarrollo y mejoramiento de las universidades son, por una parte, los proyectos del Programa para el Mejoramiento de la Calidad y la Equidad en la Educación Superior (MECESUP) y, por otra, la creación de la Comisión Nacional de Acreditación de Pregrado (CNAP). Estos procesos han inducido a la creación de unidades de estudio y planificación universitaria, lo que, sumado a las crecientes demandas de información de la educación superior, ha consolidado el desarrollo de las Direcciones de Estudios y Planificación.

Las autoridades de la Universidad de La Frontera estimaron que el trámite de la ley sobre el aseguramiento de la calidad podía demorarse. Sin embargo, se comprometieron con tales ideas asumiendo que el mejoramiento de la calidad es un factor estratégico que puede distinguirla y asegurarle su continuidad y mejor posicionamiento en el sistema universitario chileno. Han enfrentado decididamente este desafío, formulando políticas y realizando acciones en el sentido de mejorar la calidad de la formación profesional, de sus programas de posgrado y de investigación. Para ello, han generado acciones de autorregulación, tales como la implementación de procedimientos de evaluación, la revisión y actualización de normativas y procedimientos, la revisión y renovación de los planes de estudios. Se han sometido, como ya se ha afirmado, a la evaluación de pares, inicialmente en el 
contexto del Programa de Autorregulación Concordada del Consejo de Rectores y, actualmente, inspiradas en los procesos experimentales de acreditación de la CNAP.

Los primeros resultados

Hasta este momento, la Universidad de La Frontera ha acreditado seis carreras de pregrado: Medicina, Psicología, Agronomía, Ingeniería Forestal, Servicio Social e Ingeniería Civil Electrónica. Una carrera esperando dictamen: Ingeniería Civil Industrial con mención en Bioprocesos. Dos carreras esperando visitas de pares: Pedagogía en Educación Física, Deportes y Recreación, e Ingeniería Civil Industrial con mención en Informática. Cuatro carreras en última etapa de informe: Pedagogía en Historia, Geografía y Educación Cívica, Pedagogía en Castellano y Comunicación, Periodismo y Odontología. Dos carreras en proceso: Sociología y Contador Público y Auditor. Diez nuevas carreras en proceso: Kinesiología, Enfermería, Tecnología Médica, Nutrición y Dietética, Obstetricia y Puericultura, Ingeniería en Alimentos, Ingeniería Ambiental, Ingeniería Matemática, Ingeniería en Construcción, Ingeniería Comercial. En suma, 25 de sus 31 carreras.

En posgrado ha acreditado seis de sus 12 programas de magíster: Educación (tres menciones), Psicología, Epidemiología Clínica, Informática Educativa, Ciencias con mención en Morfología y Ciencias con mención en Biología de la Reproducción.

Los primeros días de agosto de 2004 el Comité de Pares Evaluadores Externos del Proceso de Acreditación Institucional realizó la respectiva Visita de Evaluación a la Universidad de La Frontera, restando sólo el dictamen del CSE.

\section{La carrera de Psicología}

Esta carrera inició sus actividades docentes en marzo de 1982, en la lógica de una universidad que decide ampliar su oferta académica. Recuérdese que la Universidad de La Frontera se crea en marzo de 1981, en Temuco, producto de la fusión de las sedes de la Universidad de Chile y de la Universidad Técnica del Estado. Se constituye así en la primera, entre las universidades regionales, en impartir esta 
formación profesional y la tercera en el ámbito nacional. Así las cosas, representa, tanto para la IX Región como para todas las regiones del sur de Chile, un significativo aporte a la formación de profesionales en psicología.

Cuenta actualmente con 250 alumnos. Permite formarse profesionalmente en las áreas de Psicología Clínica, Psicología Educacional, Psicología Laboral y Psicología Psicosocial Comunitaria. El 13\% de los docentes tiene grado de doctor, el 50\% de magíster y el $37 \%$ se encuentra en proceso de obtención de grado. Presta docencia a la mayoría de las carreras de la universidad y cuenta con una clínica psicológica que brinda servicio a la comunidad y apoya la docencia.

En este contexto, su equipo docente decide asumir el desafío de acreditación de la carrera. Lo hace con la convicción de que ello confiere garantía pública del grado en que las instituciones de educación superior cuentan con procesos eficaces para asegurar el cumplimiento de sus propósitos y objetivos y mejorar la calidad de los servicios que ofrecen, y promueve en las instituciones la existencia de políticas y mecanismos permanentes de autorregulación de la calidad en las diferentes funciones y procesos.

El proceso de autoevaluación se inició con una etapa de sensibilización y promoción al interior del Departamento de Psicología, por medio de reuniones en las que algunos docentes expusieron el fundamento del proceso, los objetivos y los criterios de evaluación. También se realizaron reuniones con docentes invitados de la carrera de Medicina de la Universidad de La Frontera, quienes ya habían experimentado el proceso; además, se celebraron reuniones informativas con los alumnos de la carrera.

Con el propósito de asumir colectivamente la tarea se incorporó la acreditación como meta de la Planificación Estratégica para el quinquenio 2000-2005. En julio del 2001 se firmó el acuerdo $N^{\circ} 69$ entre Rectoría y la Comisión Nacional de Acreditación de Pregrado. Al interior del departamento se constituyó el Comité de Autoevaluación de la Carrera de Psicología de la Universidad de La Frontera, integrado por la directora del departamento, el director de la carrera y un docente representante ante el Comité Técnico de Psicología, quienes condu- 
jeron el proceso de autoevaluación estableciendo objetivos, ámbitos de estudio y estrategias de recolección de información.

Durante los meses siguientes se realizó la recolección de información cualitativa por medio de grupos focales con docentes y alumnos de los tres ciclos de la carrera, para indagar la opinión acerca del proceso de enseñanza-aprendizaje, así como la evaluación de los alumnos respecto del desarrollo del Programa de Desarrollo Personal.

Por otra parte, se estableció coordinación con el director y secretaria de la recientemente creada Oficina de Autoevaluación y Acreditación de la Universidad, a fin de acceder a datos oficiales de la institución para completar el formulario de antecedentes.

Como una forma de involucrar a los alumnos y contribuir a la recolección de información se proyectó, desde los docentes de la carrera, la realización de tesis de grado de licenciatura en Psicología, que dio como resultado un estudio sobre la trayectoria laboral de los psicólogos egresados de la Universidad de La Frontera. Por otra parte, a través de la asignatura de pregrado "Metodología de la Investigación", los alumnos efectuaron una evaluación de la opinión de los empleadores. También, en noviembre del 2001, se aplicó el cuestionario enviado por la CNAP para obtener la opinión de los académicos de la carrera.

Durante el año 2002, la universidad, a través de la referida oficina -que centraliza la recolección de información institucional-, aportó información estandarizada a las carreras que se encontraban en proceso de autoevaluación.

A lo largo del proceso, en la medida en que se recogía la información, ésta se analizaba y discutía con grupos docentes, con el fin de elaborar y dar sentido a los datos. Una vez concluida la redacción preliminar del documento final se procedió a su socialización entre todos los académicos que dictan clases en la carrera, para lo cual se realizaron reuniones y una amplia difusión preliminar del Informe de Autoevaluación por medio de la red informática existente en el Departamento. Por último, se llevaron a cabo dos reuniones ampliadas, con todos los docentes, y cuyo objetivo fue identificar y discutir las 
fortalezas y debilidades, para posteriormente establecer el Plan de Desarrollo de la carrera de Psicología para los próximos años.

Es necesario reconocer que, en un comienzo, la recolección de información se realizó con bastante dificultad por el escaso tiempo asignado para ello, porque en las distintas dependencias de la universidad no estaba sistematizada o, simplemente, porque en una suerte de autoaprendizaje no era tan evidente la información relevante para el proceso. Una vez conformada la oficina de autoevaluación al interior de la universidad fue más fácil obtener dicha información.

A estas alturas del proceso era dable sostener que, aun cuando la identificación de las fortalezas y debilidades no se había sistematizado, la mirada crítica y autocrítica permitía discernir y juzgar y, por ende, evaluar y decidir imaginando alternativas sobre otros modos de hacer y de ser de las cosas que, ciertamente, no excluía a las personas.

Esta es la lógica que subyace al Plan de Desarrollo de la carrera de Psicología: se identifican las fortalezas y debilidades de cada uno de los seis criterios (propósitos institucionales, integridad institucional, estructura curricular, recursos humanos, infraestructura, apoyo técnico y recursos para la enseñanza, y vinculación con el medio). El paso siguiente consiste en la elaboración del Plan de Desarrollo mismo. En él se identifican las debilidades respecto de cada criterio y su respectiva Propuesta de acción, Indicador de logro, Responsable y Costo.

Tal como se había establecido, el Informe de Autoevaluacion de la carrera de Psicología fue enviado a la CNAP, instancia que designó un Comité de Pares Evaluadores que procedió a realizar la visita correspondiente con vistas a: evaluar el grado en que la carrera de Psicología de la Universidad de La Frontera se ajustaba a los criterios y parámetros establecidos; evaluar el grado de cumplimiento de los propósitos que la carrera se ha definido; y validar el Informe de Autoevaluación. Para estos efectos, se llevó acabo un proceso riguroso y sistemático de recolección, procesamiento y análisis del material pertinente.

La visita se realizó sobre la base de una agenda predefinida que consultó entrevistas con el rector; el decano de la Facultad de 
Educación y Humanidades; el Comité de Autoevaluación de la carrera; el Consejo Asesor del Departamento; el Consejo Técnico de Carrera; egresados de la carrera; el director del Departamento de Psicología; y académicos sin cargos administrativos.

El Informe Final de la Visita de Pares presenta una sinopsis de las fortalezas y debilidades identificadas. Como se trata de una carrera acreditada por cinco años, carece de sentido reproducirlas en este artículo. Sí es importante hacerlo con las recomendaciones que formula el Comité:

Diseño y aplicación de un plan de evaluación sistemática de cumplimiento de propósitos; una evaluación continúa, tanto del proceso como de los resultados de las innovaciones curriculares metodológicas, con clara indicación de indicadores que permitan tomar decisiones respecto a los ajustes necesarios; incorporar la evaluación de la confiabilidad de los criterios de admisión a los cursos del ciclo terminal, especialmente los empleados para las entrevistas de aceptación a los cursos del área clínica; fortalecer la difusión de estos criterios de admisión a los cursos del ciclo terminal y explicitarlos antes de que los estudiantes hagan sus inscripciones; desarrollar mecanismos para lograr autonomía financiera maximizando las numerosas potencialidades de sus académicos quienes aunarían esfuerzos en torno a una meta común; considerar las potenciales amenazas del entorno, especialmente las ofertas alternativas de la carrera en la región y en el resto del país.

\section{Corolario}

Para la carrera de Psicología, los logros de este proceso de acreditación están contenidos en los siguientes enunciados:

- Haber logrado sistematizar información relacionada con el hacer consustancial de la carrera.

- Haber tomado conciencia de las debilidades y fortalezas por parte de las autoridades, académicos y estudiantes.

- Generación de un movimiento proactivo al interior de la carrera.

- Regularización de los contratos. 
- Fortalecimiento del perfeccionamiento académico e investigación.

- Mejoramiento de los instrumentos de autorregulación.

Desde un punto de vista institucional, la acreditación de la carrera de Psicología -segunda carrera que lo logra, después de Medicinajunto a las que le suceden, tanto en pregrado, en posgrado como institucional, están haciendo de la acreditación "una oportunidad" para hacer una seria autocrítica. De este modo, ir generando una cultura de la calidad en una universidad que no sólo busca alcanzar mayores niveles de eficiencia y efectividad, sino que, además, hacerse responsable del rol que ha asumido en la comunidad a la que sirve. Rendir cuenta pública de ello y, sobre todo, apropiarse de la mejora continua como principio de excelencia y modo de hacer las cosas. 


\section{REFERENCIAS BiBLIOGRÁFICAS}

Block, James, Mastery Learning, Cómo aprender para lograr el dominio de lo aprendido, El Ateneo, Buenos Aires, 1975, pp. 58-64.

Coombs, P.H., El Planeamiento Educacional, Paidós, Buenos. Aires, 1970, pp. 165-166.

Gómez Millas, Juan, La constante y necesaria reforma de la Universidad, en: Estudios y Consideraciones sobre Universidad y Cultura, CPU, Santiago de Chile, 1986, pp. 52-54. 